\title{
MODAL ANALYSIS AND TESTING OF ROTATING MACHINES FOR PREDICTIVE MAINTENANCE: EFFECT OF GYROSCOPIC FORCES
}

\author{
A. Vansteenkiste; M. Loccufier; B. Vervisch; P. De Baets; \\ Ghent University, Belgium
}

\begin{abstract}
Through the year's preventive maintenance, which is based on vibration measurements has grown in importance to reduce costs. However in industry nowadays there is still a great need for good analytical prediction models that describe the dynamics of rotating structures. In this paper it is first shown how to build a model for for undamped gyroscopic systems. Secondly the model is analysed and used to make a parametric study for the design a test rig. It is found that the third and the fourth eigenfrequency changein function of the rotation speed due to the gyroscopic effect. Finally the experimental modal testing of rotating structures is discussed. The methods to excite a rotating structure in order to obtain the modal parameters are studied.
\end{abstract}

Keywords: Rotor dynamics; experimental modal testing; Campbell diagram; gyroscopic effect;

\section{INTRODUCTION}

Rotating machines are widely used in our modern life, ranging from washing machines to steam turbines and aeroplanes. Through the years there has been a tendency to faster and better performing rotating machines with lower vibration levels. However, to fulfill these design specifications, it is necessary to model, analyse and improve the dynamics of rotating structures, because the efficiency is mostly influenced by the small gaps between the rotating en the static parts. Another reason why dynamic models are needed is for predictive maintenance, which is based on vibration measurements. So, in order to evaluate those measurements it is important to have good dynamic models. However in industry there is still a great need for accurate analytical prediction models that describe the dynamics of rotating structures, such as the reliability, stability and response levels.

A problem is to obtain the parameters of the dynamical model experimentally. For non-rotating structures it is well known how to obtain the modal parameters experimentally. For rotating structures this is not the case [1].

Another problem is that it is not easy to model rotating machines analytically, as they are mostly too complex to model in a reliable way. Therefore, it is necessary to design test rigs having an easier construction and easy operating and boundary conditions. This paper shows how to model an undamped gyroscopic system and which boundary conditions and assumptions have to be made. Furthermore, a model for undamped gyroscopic systems has been built in Matlab. Simulations are done to obtain a set of parameters to design a test rig. Finally, the last part is of this paper is devoted to experimental modal testing of the test rig.

\section{MODELLING ROTATING SYSTEMS [2]}

\subsection{Assumptions for rotating systems}

A first assumption is that the vibration levels are sufficiently small such that linear models can be used. Secondly the number of degrees of freedom has to be as low as possible to limit the computation effort., Finally itis assumed that the rotating structure contains only isotropic rotating elements and that there is a quasi isotropic bearing support. Consequently the modes are planar.

\subsection{General Model for rotating systems}

The equations of motion for a rotating system, with the inclusion of the gyroscopic and the centrifugal forces have the following structure:

$$
M \ddot{q}+(G+C) \dot{q}+(K-H) q=f(t)
$$

with respectively mass matrix $M$, gyroscopic matrix $G$, damping matrix $C$, stiffness matrix $K$ and the matrix of the centrifugal forces $H$. 
Due to the gyroscopic effect, the eigenfrequencies are not all constant anymore, but some are depending on the rotating speed. This can be shown on a Campbell diagram or a whirl speed map, as for example figure 1. It can be seen that an eigenfrequency is increasing for forward whirl (FW) and decreasing for a backward whirl (BW).

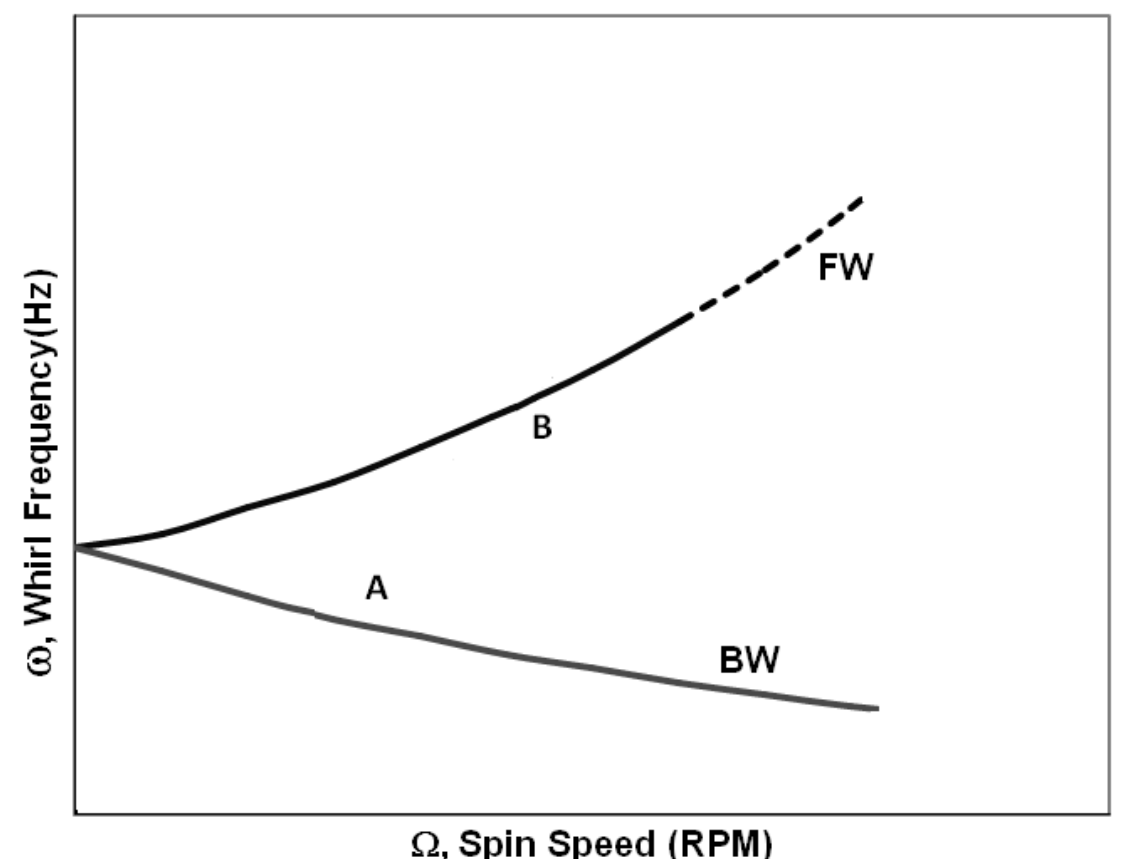

Figure 1 Campbell diagram

\subsection{Modelling undamped gyroscopic systems}

To study thethe influence of the gyroscopic effect on the vibration modes and to obtain a better insight into the gyroscopic effect and the mathematical complexity is lowered by neglecting the effect of the centrifugal force and the damping. Due to these simplifications the equation of motion is reduced to equation (2).

$$
M \ddot{q}+G \dot{q}+K q=f(t)
$$

\subsection{Finite element model of the system}

To describe the gyroscopic system, the shaft has been divided into a number of elements. The number of mass stations equals thenumber of elements +1 and is based on following concerns.

The choice of the number of elements is a compromise between enough freedom to choose excitation and measurement locations and limiting the number of modelled modes.

Figure 2 shows the chosen 10 -element model.

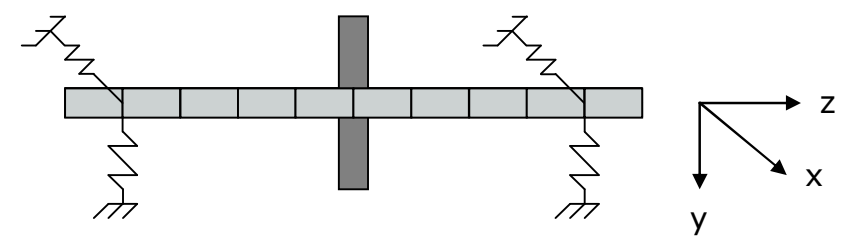

Figure 2 - finite element model with 10 elements (right-handed coordinate system)

\subsection{Obtaining matrices for a shaft finite element}

A mass station contains two linear displacements: $x$ and $y$ en two angular displacements $\theta_{x} \theta_{y}$ resulting in

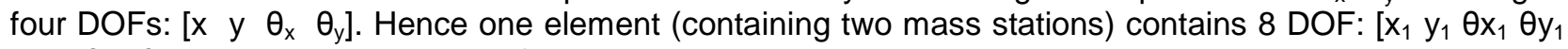
$\left.\mathrm{x}_{2} \mathrm{y}_{2} \theta \mathrm{x}_{2} \theta \mathrm{y}_{2}\right]$ which can be seen in figure 3 . 

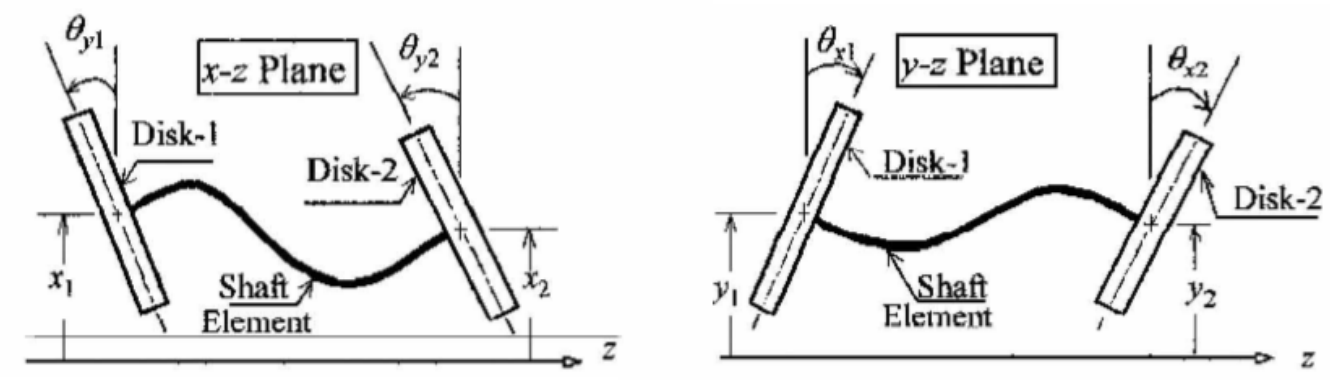

Figure 3 - Basic rotor element: angular and linear displacement in $\mathrm{x}$ - and $\mathrm{y}$-direction [2]

By using the consistent mass method, the distribution of the acceleration of the points between the nodes of the elements is given by a shape function that has the same form as a static deflection. By using the Lagrange approach the consistent mass matrix for one element can be obtained.

First the translational shaft kinetic energy of the $i^{\text {th }}$ element is calculated as equation (3) shows..

$$
T_{i}=\frac{1}{2} \cdot \frac{M_{i}}{L_{i}} \int_{0}^{L_{i}}\left(\dot{x}^{2}+\dot{y}^{2}\right) d z
$$

Substituting this into the acceleration term of the Lagrange equation (4) will yield the mass matrix.

$$
\frac{d}{d t}\left(\frac{\partial T_{i}}{\partial \dot{q}}\right)=[\mathrm{M}]^{i}\{\ddot{q}\}, \quad=1,2 \ldots 8
$$

Where $q$ denotes the vector of generalized coordinates.

The polar moment of inertia is lumped in two equal parts at the elements end points to obtain the skewsymmetric gyroscopic matrix.

The consistent stiffness matrix for one element can be similarly obtained by using the potential energy. In order to derive the $[\mathrm{M}],[\mathrm{G}]$ and $[\mathrm{K}]$ matrices for the complete free rotor, the element matrices have to be assembled. Other non-structural mass and inertia, such as that of the rotating disc can be added by lumping at mass stations. To obtain the complete $[\mathrm{K}]$ matrix of the fixed rotor, the bearing stiffness has to be added in the mass stations as well.

\subsection{Transformation into state-space form [3]}

In order to solve the generalized eigenvalue problem, the model is transformed into a state space form.

$$
x(t)=\left[\begin{array}{c}
q(t) \\
\dot{q}(t)
\end{array}\right]
$$

The equation of motion is now changed into a first order differential equation (5) or (6).

$$
\begin{gathered}
\underbrace{\left[\begin{array}{ll}
-K & \\
& M
\end{array}\right]}_{\hat{M}} \dot{x}+\underbrace{\left[\begin{array}{ll}
0 & K \\
K & G
\end{array}\right]}_{\hat{G}} x=\underbrace{\left[\begin{array}{c}
0 \\
f(t)
\end{array}\right]}_{\hat{f}(t)} \\
\hat{M} \dot{x}+\hat{G} x=\hat{f}(t)
\end{gathered}
$$

In case of a model with 10 element, this results in a model with 2.4. $(10+1)=88$ DOF.

\section{ANALYSIS AND PARAMETRIC STUDY}

In the figure 4 the different mode shapes. The two first eigenfrequencies are equal and their corresponding eigenvectors represent each a displacement in $\mathrm{x}$ - or $\mathrm{y}$-direction, that describe a spherical cylinder motion. The third and fourth eigenfrequencies are not equal anymore and their eigenvectors represent each a 
motion in $x$ - and $y$-direction, that describe a conical cylinder motion. In $x$ and $\theta_{y}$ direction the $2^{\text {nd }}$ en $4^{\text {th }}$ mode are predominant. However in $y$ and $\theta_{x}$ direction the $1^{\text {st }}$ en $3^{\text {rd }}$ mode are predominant.
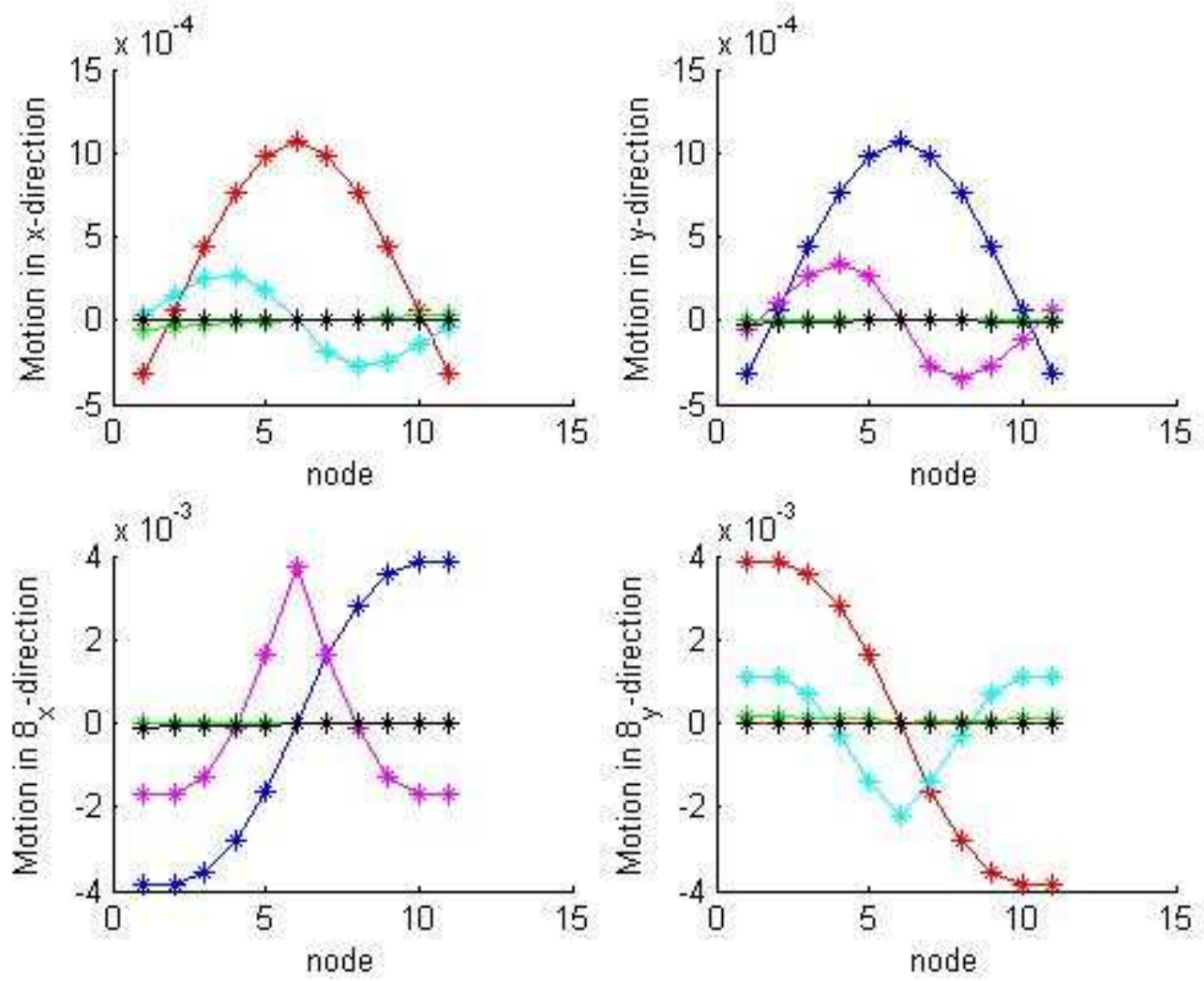

Figure 4 - Mode shapes in the nodes

To illustrate the motions, the displacement of the first node is shown in function of time in figure 5 .
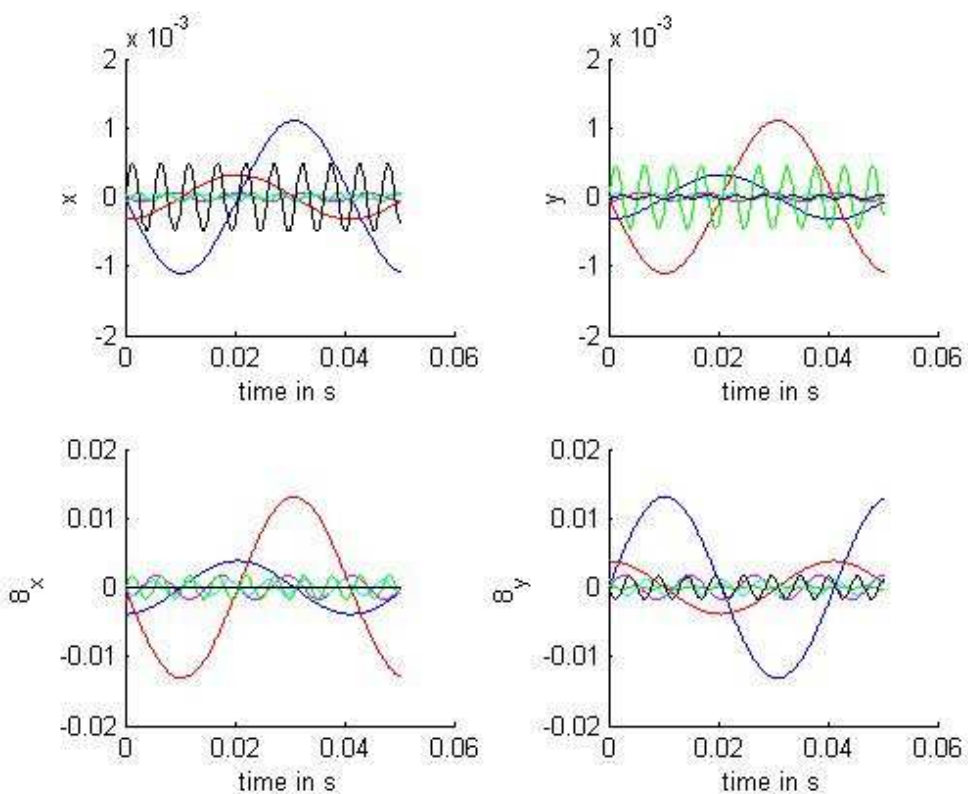

Figure 5 - Motion of the first node in function of time

To design the test rig some parameters have to be determined by simulation in Matlab. The shaft is chosen at a length of one meter. The parameters that are investigated are the shaft diameter, the mass and the dimensions of the disk, the location of the disk, the stiffness of the bearings and of course the influence of the rotating speed. The fixing method of the motor is also analysed. 


\subsection{Rotating speed}

When the rotation speed is increased, a decrease of the third eigenfrequency and an increase of the fourth eigenfrequency is observed. It can be concluded that the third and the fourth mode are depending on the rotation speed due to the gyroscopic effect. The first two eigenfrequencies are independent of the rotation speed. It can be concluded that it is preferred to have a speed range of the motor that is s sufficiently high order to see the gyroscopic effect through the difference between the third and the fourth eigenfrequency.

\subsection{Shaft thickness}

The thickness of the shaft is preferred to be as small as possible to obtain higher eigenfrequencies, which increase the gyroscopic effect. However the shaft needs a certain thickness in order to resist the bending moment due to the mass of the disk.

\subsection{Disk}

Increasing the mass moment of inertia of the disc increases the gyroscopic effect, which can be seen in a decrease of the $3^{\text {rd }}$ and an increase of the $4^{\text {th }}$ eigenfrequency. Increasing the mass of the disc lowers the eigenfrequencies and demands a stiffer shaft in order to reduce the stresses due to bending. Adding more disks is found to reduce the gyroscopic effect. It can be concluded that in order to increase the gyroscopic effect, the disk needs a high mass moment of inertia and a low mass.

\subsection{Stiffness of the bearings}

The ratio of the stiffness of the bearings to the stiffness of the shaft determines the mode shape.

\subsection{Fixation of the motor}

When the motor is fixed coupled to the shaft, this can be modelled by putting a second disk in an added node, which is not needed. To eliminate this, the motor is coupled to the shaft by a flexible coupling. The test rig will be powered by an electrical motor that will be chosen in function of the mass moment of inertia of the disk.

\section{EXPERIMENTALLY MODAL TESTING [1]}

Experimental modal testing can be divided into two steps. Firstly, there is an acquisition of response data, which is typically done by measuring the frequency response functions (FRFs). Secondly, the modal parameters are found by curve fitting of the measured response functions.

\subsection{Acquisition of the response data}

To acquire response data the test rig has to be excited. This is far more difficult than the excitation of nonrotating structures due to the rotation.

A first method is the excitation by a controlled unbalance. It is a simple method, because no special mechanical mechanisms are needed. A drawback of this method is the difficulty of maintaining a constant frequency.

A second method is the excitation with an impulse hammer. This is also an easy method because of the lack additional mechanical components. Another advantage is that several modes are excited simultaneously. However it is not easy to repeat with the same proper force and direction for several times. Another disadvantage is a lot of the noise on the signal (a high SNR).

A third method which reduces the drawbacks of the previous method is the excitation with an electrodynamic shaker that is connected by an additional bearing. With this method the force and frequency can be controlled, which permits to repeat the measurements. There is also less noise on the signal, which results in an improved quality of the signal. A drawback of this method is the additional bearing that has to be attached to the shaft and that is affected by the motion of the shaft. Thereby the force is also function of the response, which can be modelled with feedback and results in a change of the FRF.

\subsection{Obtaining the modal parameters}

The modal parameters are obtained by curve fitting, which is done in the frequency domain. The FRF has the form given in equation (7):

$$
H(\omega)=\sum_{r=1}^{N} \frac{A_{r}}{i \omega-\lambda_{r}}+\frac{\overline{A_{r}}}{i \omega-\bar{\lambda}_{r}}+E_{0}+\frac{E_{2}}{\omega^{2}}
$$


Where $E_{0}$ and $E_{2}$ are residual terms in order to compensate the modes that are out of range. For nonrotating structures the residue matrix $A_{r}$ is symmetric [3], however for rotating structures $A_{r}$ is not symmetric anymore.

\subsection{Simulation in Matlab}

With the model made in Matlab, excitations are simulated on different nodes and hence the corresponding impulse responses and FRF's (frequency response functions ) are obtained. First it can be concluded that when there is measured in the same direction as the excitation direction, there is a peak in the FRF due to the first or the second mode depending on the direction. Secondly it can be concluded that when there is measured in the other direction than the excitation direction, the third and the fourth mode peak, which is important to measure the gyroscopic effect. The highest relative peaks of the $3^{\text {rd }}$ and $4^{\text {th }}$ mode are observed when excited in the $3^{\text {rd }}$ node and measured in the $5^{\text {th }}$ node or vice versa. In figure 6 the FRF is shown for an impulse in $x$-direction applied to node 3 and measured in $y$-direction in node 5 . It can be seen that the $3^{\text {rd }}$ mode (around $84 \mathrm{~Hz}$ ) and $4^{\text {th }}$ mode (around $143 \mathrm{~Hz}$ ) are excited.

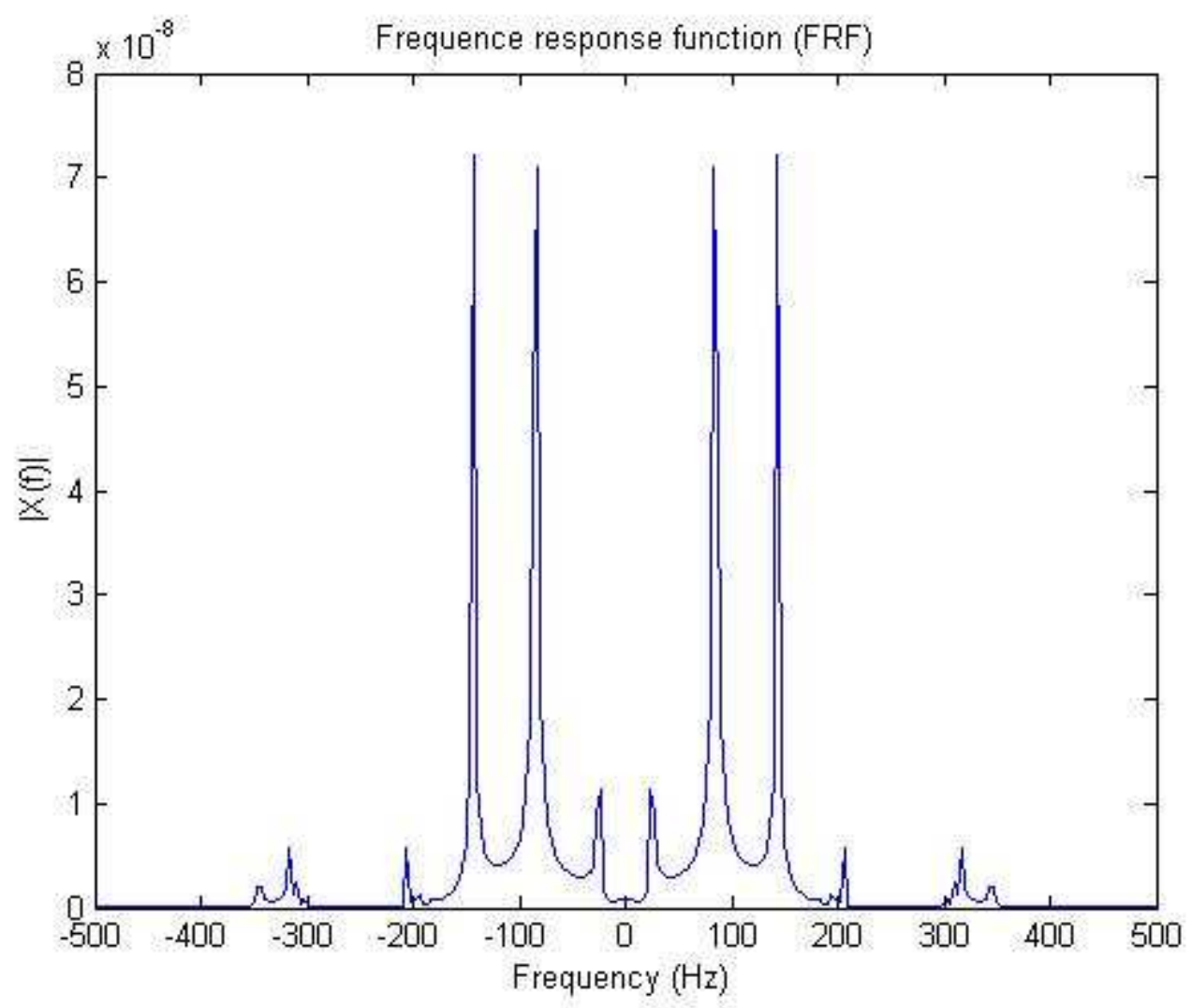

Figure 6: FRF for an impulse in node 3 (in x-direction), measured in node 5 ( in y-direction).

\section{CONCLUSION}

In this paper, a model for undamped gyroscopic systems has been built in Matlab®. Simulations have been made in order to fully understand the mechanism and to become a set of parameters to design a test rig. With those parameters a test rig will be designed in order to compare the measured modal parameters with the parameters calculated with the model in Matlab. With those results the experimental modal analysis methods can be evaluated and may be improved.

A first challenge will be to find the best excitation method. Three excitation techniques have been found in the literature and their potential and practical limitations and have been discussed. Secondly the vibrations have to be measured using accelerometers. Another difficulty will be the signal processing and the correct interpretation of the measured data.

In general it can be concluded that this topic is not yet fully known and that there are still some challenges that have to be solved before it will be possible to apply experimental modal analysis to any type of rotating machines. 


\section{NOMENCLATURE}

$M=$ mass matrix

$\mathrm{C}=$ damping matrix

$G=$ gyroscopic matrix

$\mathrm{K}=$ stiffness matrix

$\mathrm{H}=$ centrifugal force matrix

$f(t)=$ force matrix

\section{REFERENCES}

[1] Bucher I. and Ewins D.J. Modal analysis and testing of rotating structures. The royal society 2001; 359, 61-96

[2] Adams Maurice L. Rotating machinery vibration, from analysis to troubleshooting. Marcel Dekker 2000; 27-81

[3] Loccufier M. Cursus Mechanische trillingen. Universiteit Gent

[4] Brite. Development of validated structural dynamic modelling and testing techniques for vibration predictions in rotating machinery. 1996 